\title{
Optimizing Evacuation Instructions while anticipating Traveler Compliance Behavior
}

\author{
Adam J. Pel, Olga L. Huibregtse, Serge P. Hoogendoorn, Michiel C. J. Bliemer
}

\begin{abstract}
Instructing evacuees on their departure time, destination and route can lead to more efficient traffic operations. Empirical findings on evacuation behavior support the view that in practice a share of travelers decides not to comply, while current evacuation plan optimization techniques are limited to assessing mandatory evacuation under the assumption of full compliance. In this contribution we show I) how traveler compliance behavior affects evacuation efficiency, and II) how evacuation efficiency can be improved in case of partial compliance when this traveler compliance is anticipated on. The optimization method and case study application presented here underline the relevance and importance of capturing traveler compliance behavior, as this has a large impact upon the evacuation efficiency.
\end{abstract}

\section{INTRODUCTION}

$\mathrm{O}$ $\mathrm{NE}$ of the many factors determining the success or failure of an evacuation is the set-up of the evacuation plan regarding how evacuees are instructed to select their individual departure time, destination, and route. Optimizing these evacuation instructions has been studied extensively. One way to distinguish different methods to optimize instructions is by whether an evacuation traffic simulation model is used. Optimization methods which do not make use of such a traffic simulation model typically require restricting assumptions regarding, for instance, static travel times and link capacities (e.g., [1], [2]), no dynamic queuing and spillback (e.g., [3], [4]), and static network characteristics. These same assumptions are made when optimizing while using a static evacuation traffic model. These constraints clearly limit the applicability of the method to hypothesized cases. This can be regretted since the merit of these methods is faster computation, as time-consuming traffic simulations are avoided. Model-based optimization methods on the other hand exploit the simulation model to map evacuation instructions onto network outflow rates (e.g., [5]-[7]). Alternative evacuation instructions are then evaluated in an iterative manner until an (near) optimum is found. The main advantage of these model-based search methods is that more general situations can be addressed,

Manuscript received April 19, 2010.

A. J. Pel is with the Transport \& Planning Department, Delft University of Technology, Delft, The Netherlands (corresponding author, phone: +31152-784-981; e-mail: a.j.pel@tudelft.nl).

O. L. Huibregtse is with the Delft University of Technology, Delft, The Netherlands (email: o.1.huibregtse@tudelft.nl).

S. P. Hoogendoorn is with the Delft University of Technology, Delft, The Netherlands (e-mail: s.p.hoogendoorn@tudelft.nl).

M. C. J. Bliemer is with the Delft University of Technology, Delft, The Netherlands (e-mail: m.c.j.bliemer@tudelft.nl). including the impact of factors such as traffic flow dynamics and time-dependent network characteristics representing the impact of the hazard's spatial temporal evolution and prevailing traffic regulations and control.

Model-based optimization methods principally also allow including the effect of traveler compliance. This has been lacking until now even though it is occasionally mentioned as a promising future research direction. The main reason why compliance behavior is not studied lies in the fact that evacuation models which are used as prediction model are unable of modeling traveler compliance behavior. As a consequence, model-based optimization studies to date typically assume full compliance, and the evacuation operations under optimized evacuation instructions for full compliance are then presented as an upper bound for network performance. In this work, we generalize the design of optimal evacuation instructions to incorporate traveler compliance behavior. This provides insight into the impact of traveler compliance on the evacuation, and enables making the trade-off made in real-life evacuation planning between the costs associated with ensuring a higher compliance level (determined by the way in which information and instructions are deployed) and the possible benefits hereof in terms of, for instance, less congestion, lower travel times, and faster evacuation.

The setup of the paper is as follows. The next section gives an overview of the few studies in which the impact of compliance level on evacuation efficiency is evaluated, and the empirical studies which support the view on partial traveler compliance. After that, Section III briefly introduces the framework for testing the impact of (anticipating) partial compliance. This framework is then applied to a case study describing the evacuation of the Walcheren peninsula, The Netherlands, in Section IV. The final section discusses the research findings and draws some generalized conclusions.

\section{STUdiEs ON TRAVELER COMPLIANCE BEHAVIOR}

Only few studies have investigated the impact of traveler compliance on evacuation efficiency. [8] evaluates the impact of a fixed compliance rate with respect to route choice on an existing evacuation plan. In this study using VISSIM, share $x$ of the travelers complies and is thus assigned to the prescribed evacuation routes, while the remainder share of travelers, that is $1-x$, is assigned to the nearest destinations and the routes following from the userequilibrium assignment. The value of $x$ is systematically varied between 1 (full compliance) and 0 (no compliance). One of the main conclusions of the study is that lower 
compliance levels may increase evacuation efficiency since partial compliance allows some travelers to deviate to underutilized non-prescribed routes and thus compromises for flaws in the evacuation plan. The fixed compliance level and user-equilibrium assumption are relaxed in [9] where travelers' compliance behavior is modeled as an explicit trade-off between following the prescribed routes and deviating to alternative routes which are (perceived as being) more attractive at each decision point (intersection) during their trip. This route switching choice process describing traveler compliance behavior is modeled by applying a hybrid route choice model allowing for both pre-trip and enroute travel decisions. The perceived additional disutility for travelers to deviate from the instructed evacuation routes is then systematically varied, thus simulating full compliance, no compliance, and intermediate states. The conclusions on the impact hereof in case of applying straightforward evacuation instructions are in line with the findings by [8]. That is, lower compliance levels may in some cases lead to higher evacuation efficiency. On the contrary, partial compliance towards optimized instructions cannot benefit the evacuation efficiency by definition. This is illustrated in [10] by testing the impact of variations in traveler compliance levels towards optimized evacuation instructions, where the evacuation instructions were optimized based on the full compliance assumption. In the presented case study application, evacuation efficiency - measured as the number of safe arrivals within the limited amount of time available dropped by 5 to 15 percent with less than full compliance. In [9] and [10], traveler compliance behavior is modeled in a similar fashion as in this work.

The small number of studies referenced here shows how limited the research is on quantifying the impact of traveler compliance on evacuation efficiency. Yet, to the best of our knowledge, no evacuation optimization method has been proposed or applied to date in which traveler compliance behavior is anticipated on. This is unfortunate since empirical data on evacuation behavior show that the full compliance assumption is inappropriate, and that in practice a share of travelers decides not to comply ([11]-[15]). Furthermore, this study shows that evacuation efficiency can be improved in case of deploying instructions which anticipate this partial compliance.

\section{OPTIMIZATION APPROACH}

Let us first define a measure for evacuation efficiency and present the framework using the evacuation model and the optimization heuristic, after which we introduce the traffic simulation model and optimization algorithm.

\section{A. Optimization Objective and Framework}

Optimizing evacuation instructions principally means designing instructions which maximize the evacuation efficiency. Various measures of evacuation efficiency can be thought of, depending on the evacuation objective and scenario constraints. For an overview see [16] and [17].

In this work, we define evacuation efficiency, $w$, as the weighted network outflow rates integrated over time $t$,
$w(E \mid S)=\int_{0}^{T} \exp (\beta t) f(E, S, t) d t$,

adopted from [17]. The efficiency is determined by the instructions $E$ (i.e., the prescribed departure time windows, safe destinations, and evacuation routes) and on the scenario, $S$ (i.e., the hazard scenario and compliance behavior). The evacuation efficiency measure has one parameter, $\beta \geq 0$. Here, we set $\beta:=0.1$, stating that earlier arrivals are valued slightly higher than later arrivals. In other words, given that the same number of travelers successfully arrives at their destination, evacuation instructions leading to a situation in which travelers arrive earlier are considered as more efficient.

In the proposed method, we maximize the evacuation efficiency as defined by (1) by alternatingly calling the evacuation traffic simulation model which uses evacuation instructions $E$ (and traveler compliance behavior) to compute the dynamic network outflow rates $f$, and calling the optimization heuristic which uses these dynamic network outflow rates $f$ to determine better evacuation instructions $E$.

\section{B. Evacuation Traffic Simulation Model}

The evacuation model EVAQ is applied here since it incorporates traveler compliance behavior. For a detailed model description we refer to [18]. Here, we only briefly introduce how traveler compliance behavior is simulated.

First of all, the departure time compliance is modeled by assuming that the fraction $\gamma \in[0,1]$ of travelers complies and follows the instructed departure times, while the remaining travelers (equal to fraction $1-\gamma$ ) do not comply and depart at their preferred departure time. The preferred departure times are here represented by the response curve following the sigmoid curve [19] (see also Fig. 2).

Second of all, we model the destination and route compliance. Travelers are assigned to an initial prescribed destination and evacuation route upon departure, after which they choose whether or not to comply during their trip. They might deviate from the instructed evacuation route when prevailing traffic conditions are such that travelers are better off (or have the feeling of being better off) by switching to another route (possibly with another destination). Perceived route costs are computed as the route travel times and the additional disutility associated with deviating from the instructed route to an alternative route. In this work, we use prevailing travel times to model travelers' route decisions, since this is available information nowadays from most information sources, such as, radio broadcasting, variable message signs, dynamic road-side information panels, in-car navigation systems, etc. The additional disutility of noncompliance is determined by the fraction of route length of the alternative route which does not coincide with the prescribed evacuation route, and a cost term. The cost term states that the alternative route should be $\varphi$ percent faster, with a minimum of $\theta$ minutes before travelers switch routes.

The parameters $\gamma, \varphi$, and $\theta$ describe traveler compliance behavior, where $\gamma$ determines departure time compliance, while $\varphi$ and $\theta$ determine destination and route compliance. In the limiting case that $\gamma=1$ travelers depart at the instructed 
departure times indicating full compliance. On the contrary, $\gamma=0$ leads to departure times following the preferred response curve. For $0<\gamma<1$, a share of travelers complies, while the remainder of travelers does not comply. Similarly for destination and route compliance, full compliance can be modeled when high values are chosen for $\varphi$ and $\theta$. The costs of deviating from the instructed route then become very large such that all travelers comply. Noncompliance is modeled by setting $\varphi$ and $\theta$ equal to zero. The additional disutility for deviating from the prescribed destination and route then equals zero, such that travelers always follow the (perceived) fastest route, independent of which route is instructed. Partial compliance, depending on the traffic conditions, is modeled as $0<\varphi \ll \infty$ and $0<\theta \ll \infty$, where higher values allow for higher compliance rates, since travelers then require larger (travel time) gains before deviating from the instructed destination and route.

\section{Optimization Heuristic}

The optimization heuristic consists of two steps. In the first step, we generate promising instruction sets consisting of a prescribed departure time and route (where the route implies the destination). In the second step, we subsequently assign groups of travelers to these instruction sets using an ant colony optimization approach. This way, an evacuation plan is generated consisting of instructions for all travelers regarding departure time, destination and route. These evacuation plans are evaluated using the evacuation traffic simulation model. Depending on how well the plan performs, new instruction sets are selected leading to a new evacuation plan. This way, travelers are assigned to the generated instruction sets is an iterative procedure executed a large number of times in which each iteration aims at improving the current-best solution. For a detailed description of the procedure we refer to [10].

\section{MODEL APPLICATION}

In the following, we illustrate the impact of partial compliance behavior on evacuation efficiency when applying the instructions that are optimized assuming full compliance. Also, we show the impact of anticipating this partial compliance behavior and how this reduces the (negative) impact of partial traveler compliance. To this end, we will use the evacuation of the Walcheren peninsula as a case study. Let us first briefly describe the case, after which we present the experimental set-up used to structure the application, and discuss the numerical results.

\section{A. Case Description}

The Walcheren peninsula is situated in the south western part of the Netherlands and contains both rural and build-up areas (see Fig. 1). The population of approximately 120,000 inhabitants is largely concentrated in two cities. In the setting chosen here the available time to evacuate is 8 hours. After this, further evacuation is considered no longer possible due to flooding. We assume everyone prefers to depart within these 8 hours where the departure time preferences follow the sigmoid curve pictured in Fig. 2. The designated exit points are the $2 \times 2$ lane motorway, and the three $2 \times 1$ lane provincial arterials in east and northeast direction. The road network used in the analysis consists of motorways, provincial and urban arterials, and collector roads, leading to 146 links and 61 nodes, including 23 origins and 4 safe destinations (i.e., exit points).

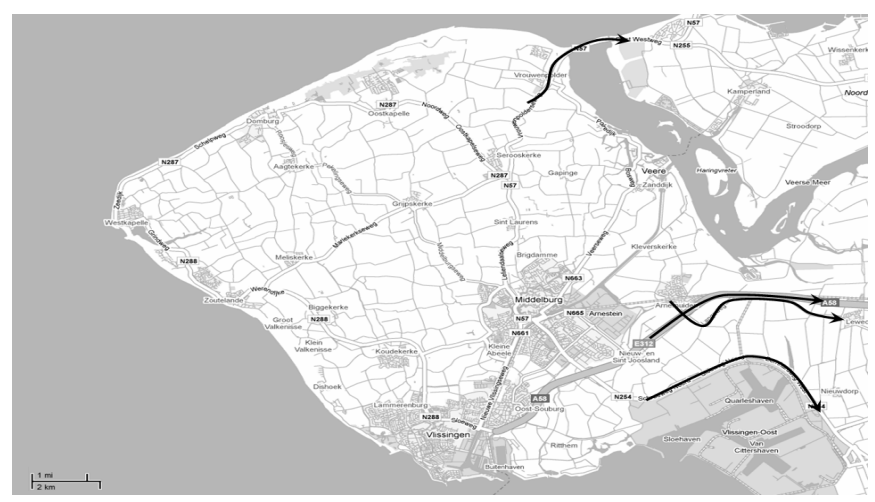

Fig. 1. Walcheren evacuation network and exit points

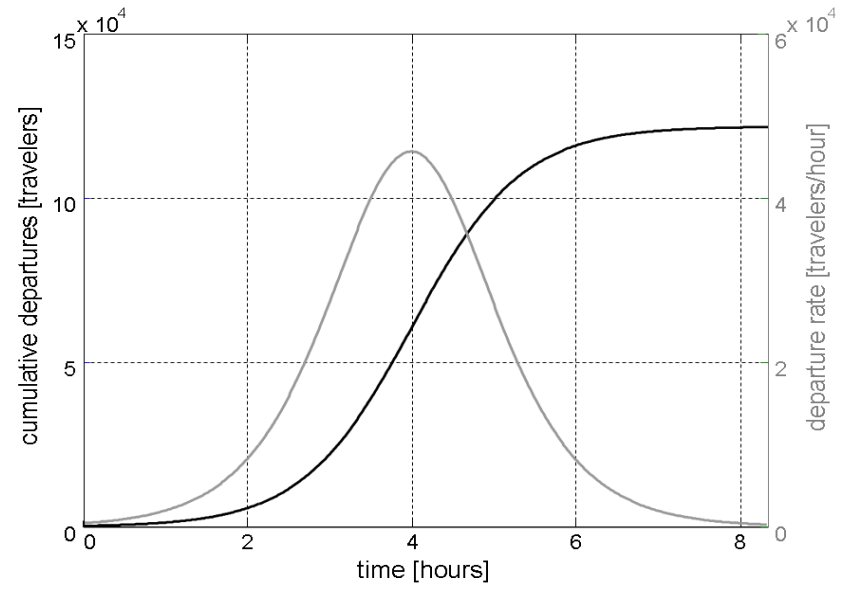

Fig. 2. Preferred departure profile

\section{B. Experimental Set-up}

The model application consists of two related parts, both showing the relevance of incorporating traveler compliance behavior in evacuation optimization. First, the (negative) impact of traveler compliance on evacuation efficiency is shown when inappropriately assuming full compliance. This is done by generating optimized evacuation instructions assuming full compliance, and then applying these optimized instructions to scenarios where the traveler compliance level is systematically varied. The departure time compliance is varied between $\gamma=1$ and $\gamma=0$, thus modeling the scenarios of full compliance, no compliance and intermediate states of partial compliance. Destination and route compliance is varied between $\theta=\varphi=\infty$ and $\theta=\varphi=0$, thereby covering all states of compliance behavior. The impact of these variations in compliance level on the evacuation operations and efficiency is then analyzed.

Second, we show the (positive) gain in evacuation efficiency when anticipating the partial traveler compliance. This is done by iteratively searching for optimal instructions while simulating (the predicted level of) traveler compliance 
behavior towards these instructions. Here, a higher and lower level of traveler compliance behavior is considered, set as respectively $\gamma=0.7, \theta=30$ minutes, and $\varphi=50 \%$ (high compliance level), and $\gamma=0.3, \theta=6$ minutes, and $\varphi=10 \%$ (low compliance level). We then compare the evacuation efficiency resulting from these instructions against that from the optimized instructions for full compliance, both applied to the case of the same partial traveler compliance level.

\section{Numerical Results}

The proposed optimization framework is implemented in Matlab. Applying a time step of 30 seconds and group size of 200 travellers (resulting in about 380 groups), CPU times on a Windows XP driven $2.8 \mathrm{GHz}$ processor range from approximately 40 to 120 seconds for computing individual scenarios. Instructions and compliance levels leading to less congested traffic conditions result in lower CPU times, due to the way in which the traffic simulation model is implemented. Also, full compliance leads to (slightly) lower CPU times since route flow rates do not need to be updated during the traffic flow propagation procedure (since travelers will not deviate from their route). Generally speaking, CPU times and memory usage are proportional to the number of unique instruction sets that travelers are assigned to, since most model variables are computed for groups of travelers with the same prescribed departure time or route.

\section{Results for Full Compliance Assumption}

Fig. 3 shows the impact of traveler compliance level on evacuation efficiency when applying instructions optimized for full compliance. The parameter settings determining traveler compliance for which this is tested are listed in Table 1. In all cases, a share of the travelers is not able to evacuate on time before the disaster causes the network to become inaccessible and the evacuation to come to a halt. As expected, a lower compliance level has a larger negative impact on the evacuation efficiency and number of arrivals.

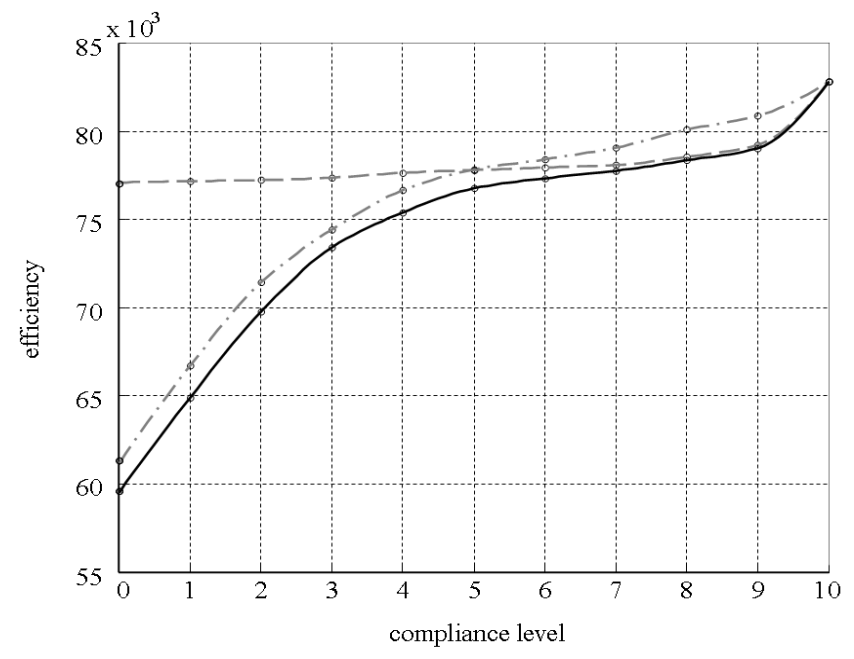

Fig. 3. Evacuation efficiency as a function of compliance level: solid line, varying departure time (DP) and destination and route (D\&R) compliance; dash-dotted line, varying DP compliance (with full compliance to $\mathrm{D} \& \mathrm{R}$ ); dashed line, varying $\mathrm{D} \& \mathrm{R}$ compliance (with full compliance to DP). Compliance levels correspond to parameter settings listed in Table 1.
Table 1. Parameter settings for compliance levels presented in Fig. 3

\begin{tabular}{cccccccccccc}
\hline & \multicolumn{1}{c}{ (non) } & \multicolumn{1}{c}{ compliance level } & \multicolumn{3}{c}{ (full) } \\
\cline { 2 - 12 } & $\mathbf{0}$ & $\mathbf{1}$ & $\mathbf{2}$ & $\mathbf{3}$ & $\mathbf{4}$ & $\mathbf{5}$ & $\mathbf{6}$ & $\mathbf{7}$ & $\mathbf{8}$ & $\mathbf{9}$ & $\mathbf{1 0}$ \\
\hline \hline $\boldsymbol{\gamma}$ & 0 & .1 & .2 & .3 & .4 & .5 & .6 & .7 & .8 & .9 & 1 \\
\hline $\boldsymbol{\theta}$ & 0 & 1 & 3 & 6 & 12 & 18 & 24 & 30 & 60 & 120 & inf \\
$\boldsymbol{\varphi}$ & 0 & 2 & 5 & 10 & 20 & 30 & 40 & 50 & 100 & 200 & inf \\
\hline
\end{tabular}

With higher compliance levels, the lower evacuation efficiency (as compared to that with full compliance) is primarily caused by noncompliance with the destination and route instructions. This is shown by the fact that an equal reduction is seen when only varying compliance towards these prescribed destinations and routes, while simulating full compliance with the departure time instructions (dashed graph). The reverse case, simulating full compliance with the destination and route instructions while varying compliance towards the prescribed departure time instructions, leads to a much smaller reduction in evacuation efficiency (dash-dotted graph). This is explained as follows.

Partial compliance leads to either under-utilized or oversaturated traffic conditions, yielding lower network outflow rates and hence evacuation efficiency. The difference is that partial traveler compliance with departure time instructions leads to all evacuation routes being under-utilized at the start of the evacuation, and being over-saturated later on. This is due to a more peaked dynamic travel demand, since with lower departure time compliance levels it more closely replicates the preferred departure profile following the sigmoid curve. Whereas partial compliance with destination and route instructions leads to some evacuation routes being under-utilized, while other routes are over-saturated, throughout the whole evacuation. That is, travelers who are instructed to follow the slower (less attractive) evacuation routes now divert to the faster (more attractive) evacuation routes. These faster evacuation routes were already critically loaded. Hence, this additional traffic flow results in oversaturated conditions. Queue spillback and rerouting behavior then cause these congested traffic conditions to spread throughout the network over time.

Below a certain compliance level, variations in destination and route compliance have only a minor impact on the evacuation efficiency. In these cases, the main cause of the additional reduction is determined by noncompliance with the departure time instructions. For these compliance levels, evacuation efficiencies for partial compliance towards departure time instructions (and full compliance with route instructions) (dash-dotted graph) is only slightly higher than for partial compliance towards both departure time and route instructions (solid graph). The reason for this is that the impact of compliance with the prescribed evacuation routes will be smaller when the network load is high, as less spare capacity is available for rerouting.

\section{E. Results for Anticipating Partial Compliance}

Anticipating the expected traveler compliance behavior while searching for optimal evacuation instructions leads to 
higher evacuation efficiency. This is shown in Table 2 which presents the evacuation efficiency as computed by (1) for the various cases. A number of findings can be made here. First of all, evacuation efficiency always increases when partial traveler compliance behavior is anticipated on, regardless of the compliance level. Second of all, this increase is larger for lower compliance levels than for higher compliance levels. This appears to be related to the fact that the impact of compliance level on evacuation efficiency is nonlinear, where lower compliance levels lead to a more than proportionate decrease in evacuation efficiency, as shown in the previous section. Hence, since the negative impact of lower compliance levels is larger, also the positive impact of anticipating these lower compliance levels can be larger. Third of all, the evacuation efficiency in case of low compliance with anticipation hereon is higher than in case of high compliance without anticipation hereon. All these three findings underline the relevance and potential of anticipating traveler compliance behavior.

Apart from these findings, two other observations can be made. First of all, evacuation efficiency resulting from optimized instructions anticipating a specific compliance level cannot increase upon lowering the compliance level. This holds by definition. However, the evacuation efficiency may (in some cases) increase upon raising the compliance level. In other words, the compliance level that maximizes the evacuation efficiency that can be gotten from a set of evacuation instructions might be higher than the compliance level that this set of instructions is optimized on. In our application, this is the case for the instructions which are optimized for the low compliance level. Second of all, although not explicitly tested here, the results shown in Table 2 suggest that evacuation instructions which are optimized for lower compliance levels might be less sensitive to variations in partial traveler compliance. This can be seen from the fact that the instructions optimized for the high (low) compliance level outperform the instructions optimized for the full compliance level when both are applied to the case of low (high) traveler compliance behavior.

Table 2. Evacuation efficiency resulting from instructions that are optimized for the full, high, and low compliance level and applied to the cases of high, and low traveler compliance behavior.

\begin{tabular}{|l||cc|}
\hline \multicolumn{1}{|c||}{} & \multicolumn{2}{c|}{ applied to } \\
\cline { 2 - 3 } optimized for & high & low \\
\hline \hline full & 77,754 & 73,411 \\
\hline high & 81,428 & 76,080 \\
\hline low & 80,817 & 80,437 \\
\hline
\end{tabular}

Recall that in the previous section it was found that the reduction in evacuation efficiency due to partial compliance levels was predominantly caused by noncompliance with destination and route instructions for high compliance levels, while predominantly caused by noncompliance with departure time instructions for low compliance levels. Not surprisingly, we see a similar pattern in the main cause for the gain in evacuation efficiency when anticipating the partial compliance level.

For high compliance levels, the gain in evacuation efficiency is primarily due to the (different) prescribed destinations and routes. This is shown Fig. 4 presenting the results for the instructions optimized for full compliance and high compliance, both applied to the case of high compliance level. Both instruction sets lead to a similar departure time profile. However, the instructions optimized for the high compliance level yield higher network outflow rates over time. The destination and route instructions which anticipate the high compliance level guide the traffic flows more efficiently such that higher network outflow rates are maintained with similar network accumulation.

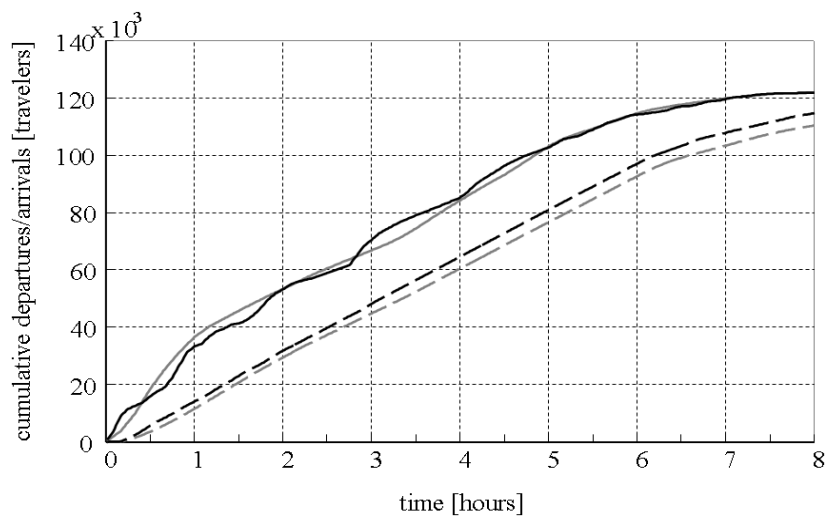

(a) Cumulative departures (solid) and cumulative arrivals (dashed)

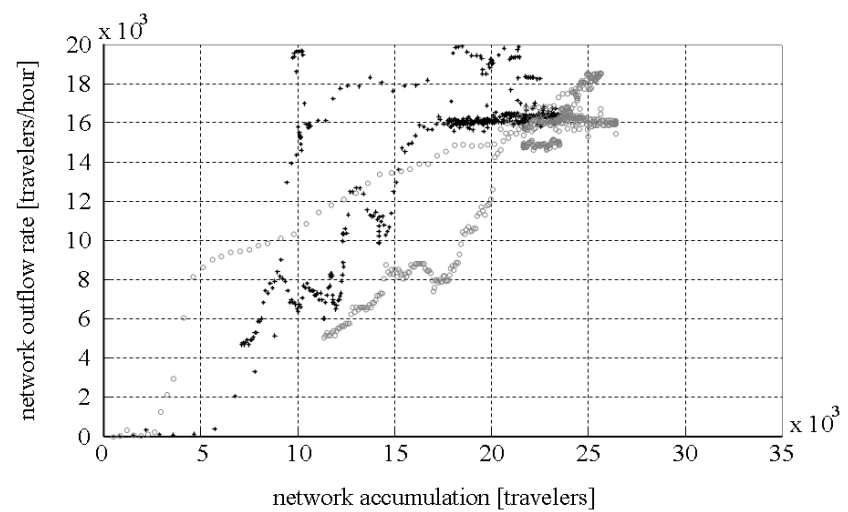

(b) Network outflow rate as a function of network accumulation (one minute averages)

Fig. 4. Model results for high compliance level: grey, not anticipating partial compliance; black, anticipating partial compliance.

For low compliance levels, this gain in evacuation efficiency is primarily due to the (different) prescribed departure times. This can be seen from Fig. 5 presenting the results for the instructions optimized for full compliance and low compliance, both applied to the case of low compliance level. Anticipating the low compliance level here leads to instructing (other) travelers to departure times letting them evacuate earlier than they would do otherwise. The larger departure rates at the start of the evacuation result in larger network outflow rates in the first few hours. Instructing these travelers to evacuate earlier not only increases the evacuation efficiency, but also limits the network accumulation later on (since these travelers move out of the peak). This is seen 
from the domains on which network accumulation is observed. The network outflow rate as a function of network accumulation shows a similar pattern for both the optimized instructions for full compliance and low compliance, thereby showing that the destination and route instructions play only a minor role here.

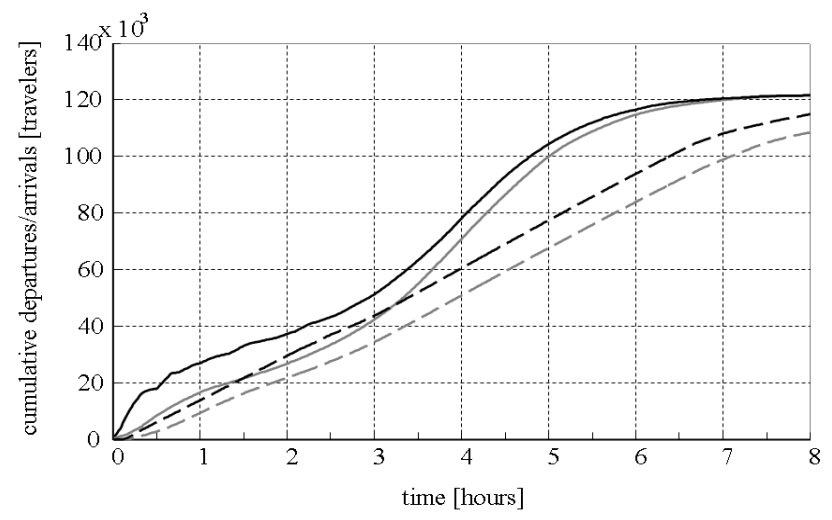

(a) Cumulative departures (solid) and cumulative arrivals (dashed)

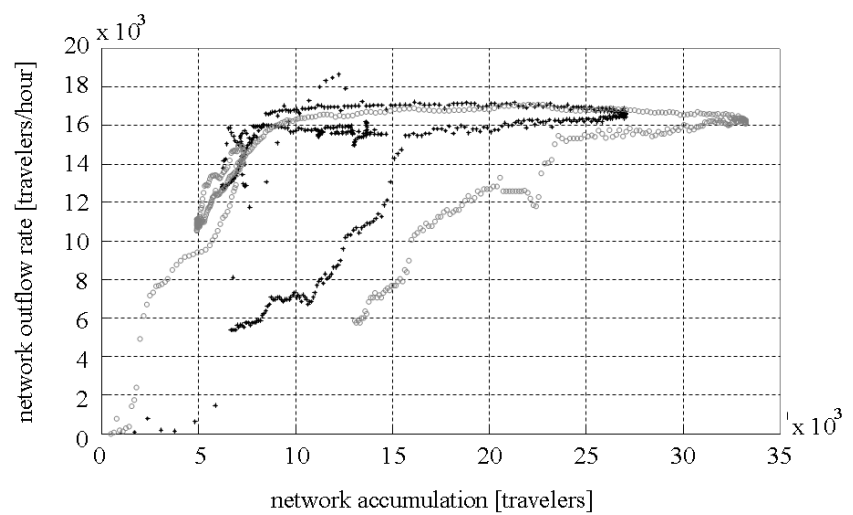

(b) Network outflow rate as a function of network accumulation (one minute averages)

Fig. 5. Model results for low compliance level: grey, not anticipating partial compliance; black, anticipating partial compliance.

\section{CONCLUSIONS}

Earlier studies on optimal evacuation instructions typically assume full compliance and present the resulting network performance as an upper bound. However, empirical data on evacuation behavior shows that in practice a share of travelers decides not to comply. In this work, we generalize the design of optimal evacuation instructions to incorporate this traveler compliance behavior. We show i) the impact of varying traveler compliance level with instructions which are optimized under the full compliance assumption, and ii) the impact of anticipating the partial traveler compliance level. The model application results illustrate how evacuation efficiency always improves when anticipating the partial compliance level, and anticipating on a low compliance level may lead to a more efficient evacuation than that of a setting of high compliance in which instructions are applied that do not anticipate on this partial traveler compliance. The findings presented here underline the relevance and potential of anticipating traveler compliance behavior, as this has shown to have a large impact upon the evacuation efficiency.

\section{REFERENCES}

[1] L. Fleisher, and M. Skutella "Quickest Flows over Time". SIAM Journal on Computing, vol. 36(6), 2007, pp. 1600-1630.

[2] Q. Lu, B. George, and S. Shekhar, "Capacity Constrained Routing Algorithms for Evacuation Planning: A Summary of Results". Lecture Notes in Computer Science, vol. 3633, 2005, pp. 291-307.

[3] N. Baumann, and E. Kohler, "Approximating Earliest Arrival Flows with Flow-Dependent Transit Times". Discrete Applied Mathematics, vo. 155,2007 , pp. 161-171.

[4] S. Opasanon, and E. Miller-Hooks, "The Safest Escape Problem". Journal of the Operational Research Society, vol. 60, 2009, pp. 1749-1759.

[5] A.M. Afshar, and A. Haghani "Heuristic Framework for Optimizing Hurricane Evacuation Operations". Transportation Research Record, vol. 2089, 2009, pp. 9-17.

[6] Y. Chiu, H. Zheng, J. Villalobos, and B. Gautam, "Modeling Nonotice Mass Evacuation using a Dynamic Traffic Flow Optimization Model". IIE Transactions, vol. 39(1), 2007, pp. 83-94.

[7] F. Yuan, and L.D. Han, "A Multi-Objective Optimization Approach for Evacuation Planning". Proceedings 1st International Conference on Evacuation Modeling and Management, The Hague, The Netherlands

[8] F. Yuan, L.D. Han, S.-M. Chin, and H. Hwang, "Does NonCompliance with Route/Destination Assignment Compromise Evacuation Efficiency?" Proceedings 86th Transportation Research Board Annual Meeting, Washington DC, US, 2007.

[9] A.J. Pel, S.P. Hoogendoorn, and M.C.J. Bliemer, "Evacuation Modeling including Traveler Information and Compliance Behavior". Proceedings 1st International Conference on Evacuation Modeling and Management, The Hague, The Netherlands, 2009.

[10] O.L. Huibregtse, S.P. Hoogendoorn, A.J. Pel, and M.C.J. Bliemer, "A Generic Method to Optimize Instructions for the Control of Evacuations". Proceedings 12th IFAC Symposium on Control in Transportation Systems, Redondo Beach, California, US, 2009.

[11] N. Dash, and B.H. Morrow, "Return Delays and Evacuation Order Compliance: The Case of Hurricane Georges and the Florida Keys". Environmental Hazards, vol. 2, 2001, pp. 119-128.

[12] M. De Jong, and I. Helsloot, "The Effects of Information and Evacuation Plans on Civilian Response during the National Dutch Crisis Exercise 'Waterproef'”. Proceedings of 1st International Conference on Evacuation Modeling and Management, The Hague, The Netherlands, 2009.

[13] K. Dow, and S.L. Cutter, "Public Orders and Personal Opinions: Household Strategies for Hurricane Risk Assessment". Environmental Hazards, vol. 2, 2000, pp. 143-155.

[14] L. Knowles, "Sydney Bushfire Emergency Evacuation: Analysis of Qualitative Research Conducted with Home Owners in Bushfireprone Areas". Working paper, Institute of Transport Studies, University of Sydney, Australia, 2003

[15] H. Rasid, H. Wolfgang, and L. Hunt, "Post-Flood Assessment of Emergency Evacuation Policies in the Red River Basin, South Manitoba". Canadian Geographer, vol. 44(2), 2000, pp. 369-386.

[16] F. Yuan, and L.D. Han, "Improving Evacuation Planning with Sensible Measure of Effectiveness Choices - A Case Study". Transportation Research Record, vol. 2137, 2009, pp. 54-62.

[17] O.L. Huibregtse, S.P. Hoogendoorn, and M.C.J. Bliemer, "Optimization of Evacuation Measures under Uncertainty". Proceedings 89th Transportation Research Board Annual Meeting, Washington DC, US, 2010.

[18] A.J. Pel, M.C.J. Bliemer, and S.P. Hoogendoorn, "EVAQ: A New Analytical Model for Voluntary and Mandatory Evacuation Strategies on Time-varying Networks". Proceedings 11th International IEEE Conference on Intelligent Transportation Systems, Beijing, China, 2008.

[19] A.E. Radwan, A.G. Hobeika, and D. Sivasailam, "A Computer Simulation Model for Rural Network Evacuation under Natural Disaster". Institute of Transport Engineers Journal, vol. 55(9), pp. 25-30 